A vibration sensor based on a distributed Bragg reflector fibre laser

This article has been downloaded from IOPscience. Please scroll down to see the full text article.

2013 Laser Phys. Lett. 10095102

(http://iopscience.iop.org/1612-202X/10/9/095102)

View the table of contents for this issue, or go to the journal homepage for more

Download details:

IP Address: 89.180.23.252

The article was downloaded on 09/08/2013 at 19:50

Please note that terms and conditions apply. 


\title{
LETTER
}

\section{A vibration sensor based on a distributed Bragg reflector fibre laser}

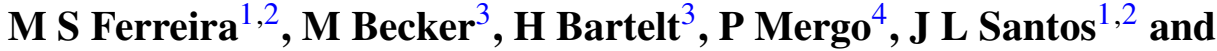 \\ O Frazão ${ }^{1}$
}

${ }^{1}$ INESC Porto, Rua do Campo Alegre, 687, 4169-007 Porto, Portugal

${ }^{2}$ Faculdade de Ciências da Universidade do Porto, Rua do Campo Alegre, 687, 4169-007 Porto, Portugal

${ }^{3}$ IPHT Jena-Institute of Photonic Technology, Albert-Einstein-Straße 9, D-07745 Jena, Germany

${ }^{4}$ Laboratory of Optical Fibres Technology, Faculty of Chemistry, Maria Curie Sklodowska University,

Sklodowska Sq 3, 20-031 Lublin, Poland

E-mail: msaf@inescporto.pt

Received 13 June 2013

Accepted for publication 17 June 2013

Published 9 August 2013

Online at stacks.iop.org/LPL/10/095102

\begin{abstract}
A vibration sensor based on a distributed Bragg reflector (DBR) is proposed. The gratings that form the cavity are written in erbium-doped fibre using the femtosecond laser technique. In this configuration, one grating of the DBR acts as a sensor whilst the other one acts as a spectral filter. The active sensor, subjected to vibration measurements, exhibits a response of up to $\sim 1.5 \mathrm{kHz}$.
\end{abstract}

(Some figures may appear in colour only in the online journal)

\section{Introduction}

The monitoring of vibrations is extremely important in areas such as civil and industrial engineering [1]. Optical fibre vibration sensors have arisen as an alternative to conventional electric ones. Different configurations have been proposed to measure vibration, based on Mach-Zehnder interferometers [2], microfibre knot resonators [3], fused-tapered optical fibre couplers [4], Michelson interferometers [5] or even fibre Bragg gratings (FBGs) [6].

On the other hand, the use of fibre lasers to perform active measurements has also been widely explored in recent years. This technology can provide a significant increase in the signal-to-noise ratio (SNR), since the amplification takes place in the optical domain, instead of the electrical one [7]. These devices have been subjected to strain [8,9], temperature [7, 10], bending [11] and twist [12].

Different fibre laser configurations have been presented to measure vibrations, from a ring fibre laser with FBGs [13, 14], to the use of a distributed feedback laser (DFB) to interrogate a serial array of Fabry-Perot interferometers [15], or even a Sagnac interferometer that was able to measure vibrations with linear high-sensitivity [16].

In this letter, a linear laser cavity is proposed to perform vibration measurements. The mirrors, two gratings written in highly doped erbium-doped fibre, are $20 \mathrm{~cm}$ apart from each other. One of the gratings is subjected to vibration measurements up to $1.5 \mathrm{kHz}$, while the other one is used as a filter.

\section{Experimental results}

The experimental setup, exhibited in figure 1, was constituted by a 980/1550 nm wavelength division multiplexer (WDM), whose input arms were connected to a $980 \mathrm{~nm}$ pump laser diode and to the data acquisition systems. The pump laser diode could achieve a maximum power of $200 \mathrm{~mW}$. Depending on the measurement carried out, an optical spectrum analyser (OSA) or a photodiode connected to an electrical signal analyser (ESA) was used. On the $1550 \mathrm{~nm}$ output arm of the WDM, a splice was made to the erbium-doped fibre. 


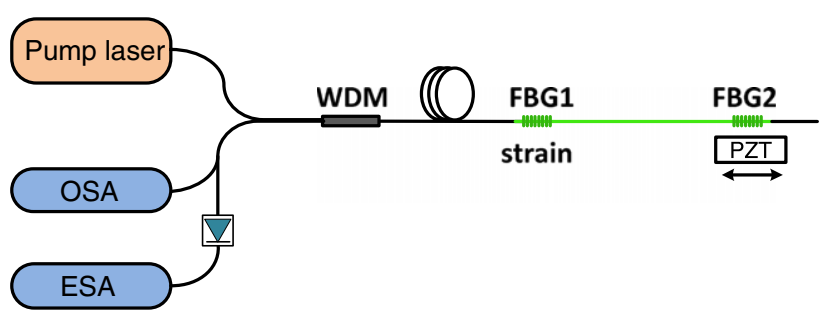

Figure 1. Experimental setup.



Figure 2. Optical spectra of the laser cavity when no strain was applied (black line), when the two FBGs were aligned (blue line) and when FBG1 was placed in the slope region of FBG2 (orange line).

In a DBR fibre laser, two FBGs enclose a rare-earth doped optical fibre section, acting as mirrors. In this case, the $\mathrm{Er}^{3+}$-doped fibre, described in [17], had a length of $\sim 20 \mathrm{~cm}$. Two fibre Bragg gratings (FBGs) were inscribed in this fibre through the femtosecond laser technique. The FBGs were written so that their Bragg wavelengths would be $\sim 1 \mathrm{~nm}$ apart from each other. Thus, the splice between the WDM output arm and the $\mathrm{Er}^{3+}$-doped fibre was made close to FBG1, which presented a lower Bragg wavelength $\left(\lambda_{\mathrm{B}}=1549 \mathrm{~nm}\right)$. FBG1 was attached to a translation stage, with a resolution of $1 \mu \mathrm{m}$, and FBG2 was attached to a piezoelectric, which was, in its turn, connected to a frequency wave generator.

With the indicated Bragg wavelength mismatch, no lasing occurred initially. However, when strain is applied to a grating, there will be a shift towards higher wavelengths (red shift). Figure 2 presents the optical spectra for different applied strains. The black line shows the initial spectra, where the first grating is read in transmission, and the second in reflection, and gain will occur in between the two. When the two Bragg wavelengths were matched, lasing emission occurred, as can be seen from the blue line of figure 2 . However, if the first grating was placed in the slope of the second one, as evidenced by the orange line in this figure, not only did the optical power increase, but also a higher sensitivity to frequency changes on the second grating was expected. When FBG2 is subjected to vibration, it will cause a small strain in the device, which will translate into an

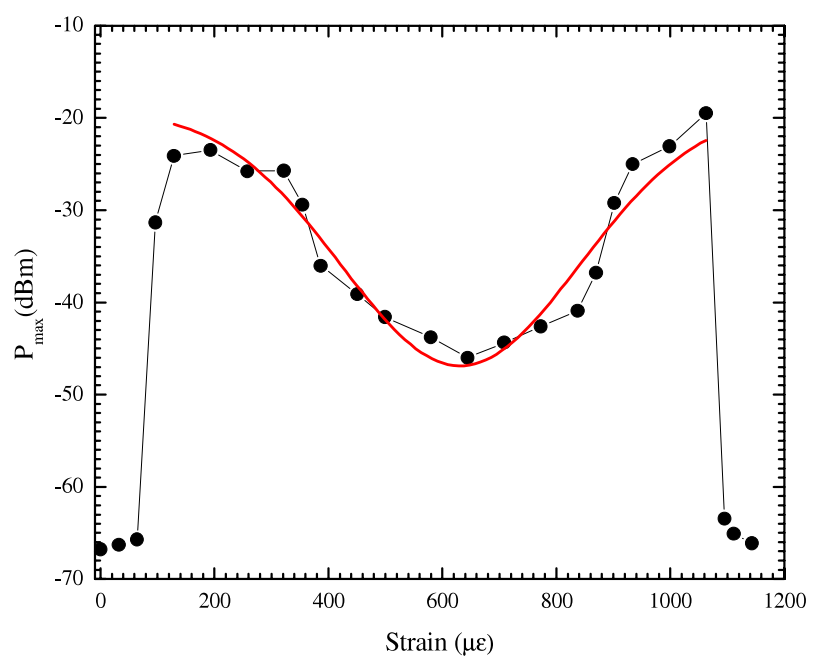

Figure 3. Laser peak power variations with applied strain. The tendency line represents the FBG spectral response.

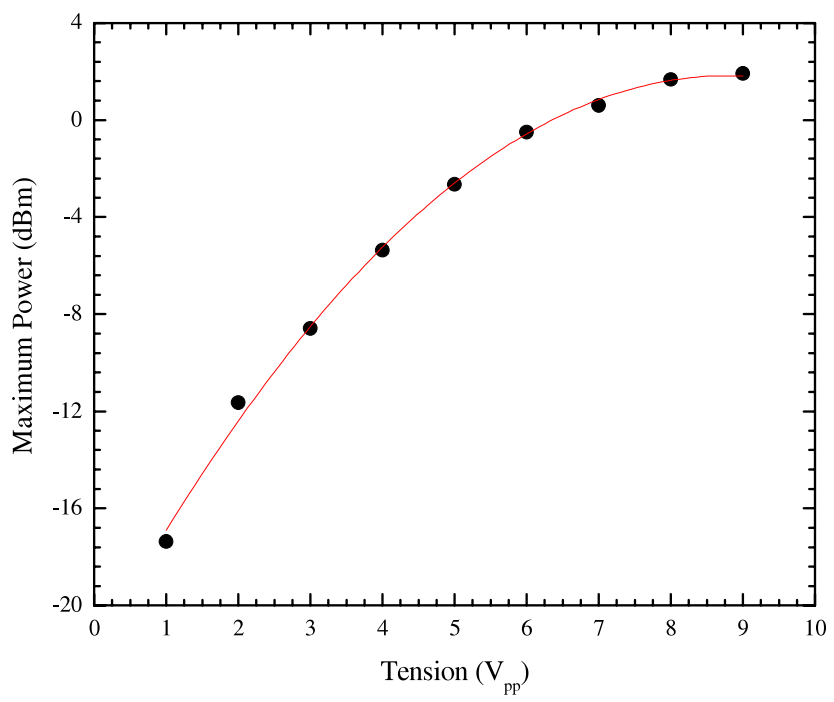

Figure 4. Dependence of the optical power on the voltage applied to the PZT.

intensity shift. This variation is expected to be observable in the frequency domain.

A thorough study of the behaviour of the laser peak power with the applied strain was performed, as can be seen in figure 3. As previously mentioned, when the Bragg wavelength of FBG1 coincides with the slope region of FBG2, there is an increase in the optical power of the laser peak. Besides, in these regions the laser only presented one emission peak, while when FBG1 was in the flat region of FBG2, several lasing peaks appeared. This multiple peak emission was due to mode hopping. In a cavity of several $\mathrm{cm}$, such as this one, the number of modes competing is extremely high, and can be determined through the cavity resonance condition $m=2 n L_{c} / \lambda_{L}$, where $n$ is the refractive index of the gain medium, $L_{c}$ corresponds to the laser cavity length, $\lambda_{L}$ is the lasing wavelength and $m$ stands for the number of modes. However, only the longitudinal modes that present sufficient gain will oscillate [7]. Once the position of the first 



Figure 5. Dependence of the average power on the sinusoidal wave frequency of the PZT for (a) the passive device and (b) the active device.

grating was established, it was held steady throughout the vibration experiments that followed. It should be noticed that the response obtained in figure 3 is not symmetrical due to the different spectral behaviour of each grating.

Frequency measurements were carried out by applying a sinusoidal wave signal to the piezoelectric. All measurements were averaged over ten samples and with a pump laser diode emission of $135 \mathrm{~mW}$. Figure 4 presents the optical power versus the peak-to-peak voltage amplitude applied to the PZT at a constant frequency of $1060 \mathrm{~Hz}$. There is a higher increase for lower tension values than for higher ones $\left(V>6 V_{\mathrm{pp}}\right)$. This is somewhat expected, since for higher tension the second grating oscillation amplitude becomes so high that it runs through the whole slope region of FBG1, thus becoming less affected by increase of the sinusoidal wave amplitude.

Setting the applied voltage at $9 V_{\mathrm{pp}}$, measurements were carried out by varying the sinusoidal wave frequency. However, the results can be misleading since the behaviour observed in figure 5(b) is a result of both the PZT response and the laser response. Thus, a characterization of the PZT was performed first, by performing the measurements without amplification in the laser cavity. As can be seen in figure 5(a), the average power increases as the frequency becomes higher. Besides, a decay of $1 / f$ in the noise can be observed. These two characteristics are due to the electronic noise of the system and the applied frequency approaching one of the PZT resonances. Figure 5(b) shows the optical power amplitude of the system with optical gain increase to an extent smaller than the one observed in figure 5(a). This may be due to the combination of the modulated grating starting to approach the non-linear region of FBG1 and the non-linear effects associated with laser cavity operation.

\section{Conclusions}

In summary, a vibration sensor based on a distributed Bragg reflector was presented. The two fibre Bragg gratings were written in erbium-doped fibre using a femtosecond laser. One of the gratings was subjected to frequency variations. The active sensor exhibited a response of up to $\sim 1.5 \mathrm{kHz}$. Usually, the whole structure of a DBR fibre laser is subjected to the measurand, when used as a sensor. In this case, one of the gratings was subjected to vibration and the other one acted as a filter. This laser cavity is easy to fabricate and exhibits good response to vibration, proving to be a good choice to perform active vibration measurements. Besides, it can also be used for remote sensing or as a multiplexable system.

\section{References}

[1] Perrone G and Vallan A 2009 A low-cost optical sensor for noncontact vibration measurements IEEE Trans. Instrum. Meas. 58 1650-6

[2] Hernández-Serrano A I, Salceda-Delgado G, Moreno-Hernández D, Martínez-Ríos A and Monzón-Hernández D 2013 Robust optical fiber bending sensor to measure frequency of vibration Opt. Lasers Eng. 51 1102-5

[3] Sulaiman A, Muhammad M Z, Harun S W, Arof H and Ahmad H 2013 Demonstration of acoustic vibration sensor based on microfiber knot resonator Microw. Opt. Technol. Lett. 55 1138-41

[4] Chen R, Fernando G F, Butler T and Badcock R A 2004 A novel ultrasound fibre optic sensor based on a fused-tapered optical fibre coupler Meas. Sci. Technol. 15 1490-5

[5] Hong X, Wu J, Zuo C, Liu F, Guo H and Xu K 2011 Dual Michelson interferometers for distributed vibration detection Appl. Opt. 50 4333-8

[6] Wada A, Tanaka S and Takahashi N 2012 Optical fiber vibration sensor using FBG Fabry-Perot interferometer with wavelength scanning and Fourier analysis IEEE Sens. J. 12 225-9

[7] Mandal J, Shen Y, Pal S, Sun T, Grattan K T V and Augousti A T 2004 Bragg grating tuned fiber laser system for measurement of wider range temperature and strain $O p t$. Commun. 244 111-21

[8] Ball G A, Meltz G and Morey W W 1993 Polarimetric heterodyning Bragg-grating fiber-laser sensor Opt. Lett. 18 1976-8

[9] Beverini N, Maccioni E, Morganti M, Stefani F, Falciai R and Trono C 2007 Fiber laser strain sensor device J. Opt. A: Pure Appl. Opt. 9 958-62

[10] Mandal J, Pal S, Sun T, Grattan K T V, Augousti A T and Wade S A 2004 Bragg grating-based fiber-optic laser probe for temperature sensing IEEE Photon. Technol. Lett. $16218-20$

[11] Liu W, Guo T, Wong A-C, Tam H-Y and He S 2010 Highly sensitive bending sensor based on $\mathrm{Er}^{3+}$-doped DBR fiber laser Opt. Express 18 17834-40 
[12] Wo J, Jiang M, Malnou M, Sun Q, Zhang J, Shum P P and Liu D 2012 Twist sensor based on axial strain insensitive distributed Bragg reflector fiber laser Opt. Express $202844-50$

[13] Tanaka S, Ogawa T, Thongnum W, Takahashi N and Takahashi S 2003 Thermally stabilized fiber-Bragg-grating vibration sensor using Erbium-doped fiber laser Japan. J. Appl. Phys. 42 3060-2

[14] Tsuda H 2010 Fiber Bragg grating vibration-sensing system, insensitive to Bragg wavelength and employing fiber ring laser Opt. Lett. 35 2349-51
[15] Manuel R M, Shlyagin M G, Miridonov S V and Meyer J 2012 Vibration disturbance localization using a serial array of identical low-finesse fiber Fabry-Perot interferometers IEEE Sens. J. 12 124-7

[16] Rivera J L, Sánchez M P, Miridonov A and Stepanov S 2013 Adaptive Sagnac interferometer with dynamic population grating in saturable rare-earth-doped fiber Opt. Express 21 4280-90

[17] Ferreira M S, Santos J L, Mergo P and Frazão O 2013 Torsion sensor based on a figure-of-eightcavity fibre laser Laser Phys. Lett. 10045105 